\title{
A proof-of-concept study on the use of prostate artery embolization prior to definitive radiotherapy in prostate cancer
}

\author{
Jeffrey Peacock \\ H Lee Moffitt Cancer Center and Research Institute https://orcid.org/0000-0002-0725-5925 \\ Dhiraj Sikaria \\ USF Health Morsani College of Medicine \\ Laura Maun-Garcia \\ H Lee Moffitt Cancer Center and Research Institute \\ Khosrow Javedan \\ H Lee Moffitt Cancer Center and Research Institute \\ Kosj Yamoah \\ H Lee Moffitt Cancer Center and Research Institute \\ Nainesh Parikh ( $\square$ Nainesh.parikh@moffitt.org) \\ Interventional Radiology, Moffitt Cancer Center, Florida, USA https://orcid.org/0000-0001-7563-8605
}

\section{Research}

Keywords: Prostate artery embolization, Prostate Cancer, Radiation

Posted Date: June 22nd, 2020

DOI: https://doi.org/10.21203/rs.3.rs-36761/v1

License: @ (i) This work is licensed under a Creative Commons Attribution 4.0 International License. Read Full License

Version of Record: A version of this preprint was published at Advances in Radiation Oncology on May 1st, 2021. See the published version at https://doi.org/10.1016/j.adro.2020.11.004. 


\section{Abstract}

Backgrounds: Prostatic artery embolization (PAE) has been well studied as a clinically effective therapy for alleviating lower urinary tract symptoms (LUTS) in patients with benign prostatic hyperplasia (BPH). We demonstrate a proof of concept for PAE prior to definitive radiotherapy in patients with prostate cancer.

Methods: From 12/2017 to 07/2019, 57 patients underwent PAE for LUTS and BPH. Nine of these patients had PAE for LUTS in the setting of localized prostate cancer prior to receiving radiation. Five of the nine patients received their entire radiotherapy course at our institution and had clinical follow up were included in the analysis. Median follow up was 18 months from the time of PAE. LUTS improvement quantified by IPSS was the primary endpoint and a two tail students $T$ test was used to compare statistical significance. Side effects during radiation were quantified using the CTCAE scoring system. Pre- and post- PAE plans were compared in the five patients that by performing an isovolumetric expansion of the post PAE plan (treated plan) equivalent to the measured volume reduction after PAE. Patient 1 and 2 had prostate and seminal vesicle RT alone while patients 3-5 had prostate with elective nodal coverage. Mean doses to organs at risk were compared between the two plans.

Results: The average IPSS score pre-PAE was 17.40 compared to post-PAE of $3.6(p=0.02)$. The average IPSS score reduction after PAE was 13.8 (5-30). The average prostatic volume reduction after PAE was $23.14 \%$ (7.2\% - 47.7\%). There were no CTCAE grade 3 (severe) or higher during radiation treatment. Post-PAE plans in patient 1 and 2 had on average $16.7 \%$ and $39.8 \%$ decrease in mean dose across the bladder, rectum, and penile bulb compared to the pre-PAE plans. There were no appreciable differences in dosimetry in the patients $3-5$ who had nodal coverage. There was no biochemical failure in any of the patients.

Conclusion: We demonstrate a proof of concept that prostate artery embolization is useful as an adjunctive procedure to alleviate LUTS, achieve significant volume reduction prior to radiation therapy, and decrease radiation related toxicity in the treatment of prostate cancer.

\section{Background}

Lower urinary tract symptoms (LUTS) due to benign prostatic hyperplasia (BPH) are a significant cause of morbidity and healthcare expenditure. Mainstays of BPH medical therapy include 5-alpha reductase inhibitors or alpha- 1 blockers although patients are often refractory for several reasons. ${ }^{1}$ The gold standard for moderate-severe LUTS from BPH is transurethral resection of the prostate (TURP). However, TURP is associated with many possible post-operative complications including sphincter injury, infection, retrograde ejaculation, bladder neck stenosis, erectile dysfunction, and TURP syndrome. ${ }^{2}$

Prostatic artery embolization (PAE) has recently been well-studied as a minimally invasive alternative to TURP, particularly for patients with "massive" glands (i.e. glands > $80 \mathrm{gm}$ ). ${ }^{3-5}$ PAE has been shown to be associated with shorter hospital stays and lower cost when compared directly to TURP, with equivalent clinical outcomes. ${ }^{6-9}$ Additionally, PAE has been associated with significant prostate volume reduction of up to $40 \% .{ }^{6}$ Thus PAE is a safe and effective treatment alternative for patients with moderate-severe LUTS from $\mathrm{BPH}^{10}$ and in some clinical situations, may in fact be preferred.

The utility of PAE in patients with prostate cancer is still in its early stages of investigation. Palliative use of PAE in a small set of symptomatic patients with inoperable prostate cancer was associated with significant LUTS improvement at 1 month. ${ }^{11}$ In a set of 12 prostate cancer patients who underwent PAE six weeks before radical prostatectomy, analysis of the pathologic prostate specimens demonstrated tumor regression and necrosis in some areas of the prostate, associated with viable tumor cells in other area of the gland. ${ }^{12}$ Therefore PAE currently may best be thought of as an adjunctive therapy rather than monotherapy.

The benefit of PAE prior to definitive radiotherapy in men with localized prostate cancer has not been published until this analysis. Radiation is considered standard of care for prostate cancer, but can result in exacerbation of LUTS during treatment, with acute or chronic toxicity ranging from $10-25 \% .{ }^{13}$

We investigate the advantages that PAE may confer to controlling symptoms prior to radiation. We also investigate the effect of post-PAE volume reduction on reduction of radiation dose to organs at risk. Finally, we suggest the concept that PAE prior to radiotherapy may lead to decreased toxicity.

\section{Methods}

This is an IRB approved retrospective study that evaluated 57 patients with the diagnosis of prostate cancer received PAE for moderatesevere LUTS from 12/2017 to 07/2019. In nine of these patients, PAE was performed at least 6 weeks prior to definitive radiotherapy for the 
treatment of their prostate cancer. Only patients who had their entire radiotherapy treatment session at our institution and who had 6 months follow up were included in our study $(\mathrm{N}=5)$. The other patients either did not complete RT at the time of the analysis or received their treatment at an outside facility. Thus, a total of 5 patients were thus included in the analysis. Median patient age was 71 years (48-76) and four of the five patients were considered to have high-risk prostate cancer. Median follow up was 18 months from the time of PAE. The average pre-PAE prostate volume size was 95.82cc (33.6-231cc). The average pre-PAE IPSS score was 17.40 (7-35). Patients 1 and 2 received prostate and seminal vesicle radiation alone. Patients 3,4 , and 5 had radiation to the pelvis for elective nodal coverage in addition to the prostate and seminal vesicles. Radiation followed the PAE procedure with a median time of approximately 4.5 months (2-14.5months).

PAE was performed by a single interventional radiologist. Femoral or radial access was obtained, a 4 or $5 \mathrm{Fr}$ base catheter was used to access each internal iliac artery and a $2.4 \mathrm{Fr}$ microcatheter was used to access each prostatic artery (see Figure 1). Embolization was performed using 300-500 micron embospheres in a dilute mixture (Merritt Medical, Inc., Salt Lake City, UT). All patients were admitted for overnight observation post procedure and were given a standard 7-day post-procedural cocktail of prophylactic medications.

Society of Interventional Radiology (SIR) grading scale was used to assess complications from PAE. IPSS score as used to assess clinical response to PAE at 3 months after the procedure and Common Terminology Criteria for Adverse Events CTCAE was used to assess GI/GU toxicity during radiation treatment. Changes in IPSS were compared using a two tailed Student's T-test.

Pre-PAE prostate volumes were contoured on T1/T2 MRI image sequences that were acquired prior to PAE by a radiation oncologist on the Pinnacle treatment planning software. Post-PAE prostate volumes were determined at the time of CT simulation for radiation therapy.

In order to identify the dosimetric differences between the pre- and post- PAE volumes, the five patients had plans created using the pre-PAE prostate volumes. The pre-PAE PTV was simulated by creating an isovolumetric expansion equal to the change in prostate volume prior to PAE and at the time of CT simulation (see Figure 2). Five pre-PAE plans were created by a dosimetrist specializing in prostate cancer radiation treatment plans blinded to the intent of the research. The pre-PAE PTV were compared to the actual treatment plans (post-PAE PTV) that the patient received during treatment. In the event of nodal volume coverage, only the prostate PTV contours were expanded and elective nodal volumes were the same for the pre and post PTV plans. The organ at risk dose was assessed using mean dose to the rectum, bladder, and penile bulb.

\section{Results}

Patient characteristics are described in Table 1. The volume reduction from the PAE was $23.14 \%$ (7.2\%-47.7\%). The average IPSS score prePAE was 17.40 compared to post-PAE score of $3.6(p=0.02)$. The average IPSS score reduction after PAE was 13.8 (5-30).

The indications for PAE were for symptomatic improvement of LUTS prior to definitive radiotherapy in all cases. There was no SIR grade C (require therapy or hospitalization $>48$ hours) or higher side effects of the procedure. There were no CTCAE grade 3 (severe or medically significant) or higher during radiation treatment. No patients had biochemical failure with a median follow up of 18 months.

Dosimetric differences were compared between patient 1 and 2 that had prostate and seminal vesicle treatment alone are shown in Figure 3 . Raw values and percent difference in estimated dosage for the two patients are given in Table 2 . The PTV covered by the $100 \%$ prescription isodose line in Patient 1 and 2 is slightly higher in the post PAE plan compared to the pre PAE plan (98.51\% vs $94.6 \%$ and $99.5 \%$ vs $98.2 \%$, respectively). Despite maintaining adequate coverage, there is a large difference in mean rectal, penile bulb, and bladder dose between the pre and post PAE plans in favor of lower dose to both post PAE plans. Post-PAE plans in patient 1 and 2 had on average $16.7 \%$ and $39.8 \%$ decrease in mean dose across the bladder, rectum, and penile bulb, compared to the pre-PAE plans. There were no appreciable dosimetric differences in patients 3,4 , and 5 who had elective nodal coverage for their high risk prostate cancer.

Table 1 - Patient and treatment characteristics. 


\begin{tabular}{|c|c|c|c|c|c|c|c|c|c|c|c|c|c|c|}
\hline & Age & $\begin{array}{l}\text { Date of } \\
\text { PAE }\end{array}$ & $\begin{array}{l}\text { Pre- } \\
\text { vol }\end{array}$ & $\begin{array}{l}\text { Post } \\
\text { vol } \\
\text { in } \\
\text { cc }\end{array}$ & $\begin{array}{l}\% \\
\text { Difference }\end{array}$ & $\begin{array}{l}\text { Pre- } \\
\text { IPSS }\end{array}$ & $\begin{array}{l}\text { Post- } \\
\text { IPSS }\end{array}$ & ADT & Gleason & $\begin{array}{l}\text { T } \\
\text { stage }\end{array}$ & $\begin{array}{l}\text { Pre- } \\
\text { PSA }\end{array}$ & $\begin{array}{l}\text { Post- } \\
\text { PSA }\end{array}$ & Radiation & $\begin{array}{l}\text { Delta in } \\
\text { months }\end{array}$ \\
\hline $\begin{array}{l}\text { PT- } \\
01\end{array}$ & 48 & $9 / 2017$ & 231 & 165 & $28.6 \%$ & 7 & 2 & $Y$ & $3+3$ & T1c & 37.7 & 7.25 & $3 G y \times 20$ & 10 \\
\hline $\begin{array}{l}\text { PT- } \\
02\end{array}$ & 71 & $12 / 2018$ & 153 & 80 & $47.7 \%$ & 35 & 5 & $\mathrm{~N}$ & $4+3$ & T1c & 55.9 & 3.18 & $2 \mathrm{~Gy} \times 39$ & 3 \\
\hline $\begin{array}{l}\text { PT- } \\
03\end{array}$ & 73 & $01 / 19$ & 126 & 105 & $16.7 \%$ & 18 & 6 & $Y$ & $4+4$ & T1c & 6.4 & 0.44 & $2 \mathrm{~Gy} \times 29$ & 14.5 \\
\hline $\begin{array}{l}\text { PT- } \\
04\end{array}$ & 76 & $1 / 2018$ & 33.5 & 31.1 & $7.2 \%$ & 13 & 4 & $Y$ & $4+5$ & T3 & 0.46 & 0.04 & $\begin{array}{l}1.8 \mathrm{~Gy} x \\
45\end{array}$ & 2 \\
\hline $\begin{array}{l}\text { PT- } \\
05\end{array}$ & 68 & $3 / 2018$ & 116 & 98 & $15.5 \%$ & 14 & 1 & $Y$ & $3+4$ & T1c & 2.09 & 1.69 & $\begin{array}{l}15 \mathrm{~Gy} \\
\text { brachy } \\
+1.8 \mathrm{~Gy} x \\
25\end{array}$ & 4.5 \\
\hline
\end{tabular}

Table 2 - The coverage and mean dose to OAR using pre-and post-PAE volumes from two separate plans.

\begin{tabular}{llll} 
Patient 1 & Pre-PAE & Post-PAE & \% difference \\
\hline Volume cc & $231 \mathrm{cc}$ & $165 \mathrm{cc}$ & $\mathbf{- 2 8 . 5 7 \%}$ \\
\hline PTV coverage \% & $94.6 \%$ & $98.51 \%$ & \\
\hline Bladder dose & $2267.1 \mathrm{cGy}$ & $1914.2 \mathrm{cGy}$ & $\mathbf{- 1 5 . 5 7 \%}$ \\
\hline Penile Bulb dose & $2569.5 \mathrm{cGy}$ & $2110.4 \mathrm{cGy}$ & $\mathbf{- 1 7 . 8 4 \%}$ \\
\hline Rectum dose & $3205.6 \mathrm{cGy}$ & $2667.7 \mathrm{cGy}$ & $\mathbf{- 1 6 . 7 8 \%}$ \\
\hline Patient 2 & & & \\
\hline Volume cc & $153 \mathrm{cc}$ & $80 \mathrm{cc}$ & $\mathbf{- 4 7 . 7 1 \%}$ \\
\hline PTV coverage \% & $98.2 \%$ & $99.5 \%$ & \\
\hline Bladder dose & $2787.2 \mathrm{cGy}$ & $1926.6 \mathrm{cGy}$ & $\mathbf{- 3 0 . 8 7 \%}$ \\
\hline Penile Bulb dose & $4355.2 \mathrm{cGy}$ & $3142.0 \mathrm{cGy}$ & $\mathbf{- 2 7 . 8 5 \%}$ \\
\hline Rectum dose & $3554.8 \mathrm{cGy}$ & $\mathbf{1 3 9 9 . 3 \mathrm { cGy }}$ & $\mathbf{- 6 0 . 6 4 \%}$
\end{tabular}

\section{Discussion}

This study confirms the proof of concept that PAE reduces the rate of GU toxicity after definitive radiotherapy for prostate cancer in patients who have LUTS. All patients in this study experienced significant improvement in LUTS at 3 months after PAE, which is consistent with previously published results. ${ }^{6-9}$ Moreover, despite these patients prior to PAE having significant LUTS that would predispose them to acute toxicity during radiation, no patient experienced grade 3 or higher toxicity during radiation treatment.

While the notion that acute GU toxicity improves significantly by having PAE prior to RT, the additional benefit of prostate volume reduction also has a significant impact on treatment planning. We demonstrate in two cases that PAE resulted in a significant reduction in OAR dose. Numerous randomized studies have shown that reducing dose to OAR reduces toxicity in prostate radiation. The transition from 2D to 3DCRT for example has been shown to improve disease control by escalating dose while improving radiation related side effects with decreased dose to normal tissue. ${ }^{14}$ This benefit in the therapeutic ratio parallels the benefits that PAE may offer in well selected patients. By reducing the volume of the prostate one can effectively treat the disease and limit dose to normal tissue.

Of note the remaining three patients who had nodal volume coverage did not see any appreciable differences in OAR dosing when treating to the pre and post-PAE PTV volumes since the entire pelvis is treated followed by a boost to the prostate. The changes in pre-PAE PTV only impacted the boost volume. We therefore conclude that most of the benefit will be seen in patients who are treated to the prostate and or seminal vesicles alone. In those cases, PAE can be a useful adjunctive therapy by allowing for higher dose while simultaneously resulting in decreased short- and long-term GU/GI toxicity. 
For well selected patients who are treated with localized prostate cancer, stereotactic body radiation therapy (SBRT) is an increasingly popular modality of ultra-hypofractionation. Often these regimens allow for the completion of radiation therapy in as little as $3-5$ days. The volume of treatment as it relates to OAR proximity is often the limiting factor when considering viability of SBRT. As such, the volume reduction achieved by PAE may well be successful for providing patients with more convenient treatment modalities such as SBRT.

All of the patients analyzed showed clinical improvement after PAE based on significant IPSS improvement. Based on this study, PAE has a significant potential to decrease morbidity. Furthermore, the results show that the benefits of PAE as a minimally invasive method for control of LUTS can be extended to the prostate cancer population without adverse results. For example, PT-04 was reliant on straight catheterization prior to PAE, but was able to void without catheterizations 12 weeks after PAE.

\section{Limitations}

Isovolumetric expansion may not accurately reflect treatment to the pre-PAE volume. It was used as the best approximation to compare the pre and post-PAE volumes in treatment planning. This study also has a low patient volume and demonstrates a proof of concept. Additionally, there is a theoretical radiobiological concern with radiation after PAE. Hypoxia is a known mechanism of radioresistance, which may be induced within the tumor after PAE similar to embolization for hepatocellular carcinoma. ${ }^{15}$ It is unclear if PAE impacts the efficacy of radiotherapy due to hypoxic effects. However, it is clear that the predominance of the ischemic and necrotic effects after the embolization take place in the central gland. ${ }^{11}$ Conversely, the majority ( $\left.85 \%\right)$ of prostate cancer lesions occurs in the peripheral zone, thus potentially negating the thought that hypoxia after PAE might lead to radioresistance. Indeed, there has been no biochemical failure in any of the patients in this study, but longer term follow up with more patients is required. However, the symptomatic and dosimetric benefit is apparent and warrants further investigation, particularly in patients who receive prostate and seminal vesicle radiation alone.

\section{Conclusion}

We demonstrate provide a proof of concept study showing that PAE is an effective adjunctive therapy for improving LUTS and achieving significant volume reduction prior to definitive radiation in prostate cancer patients. This finding demonstrates that PAE prior to definitive radiotherapy decreases doses to OAR, which may result in clinically significant decrease in radiation-induced toxicity.

\section{Abbreviations}

PAE - prostate artery embolization, OAR - organ at risk, SBRT - stereotactic body radiation therapy, LUTS - lower urinary tract symptoms, $\mathrm{BPH}$ - benign prostatic hyperplasia, TURP - transurethral resection of the prostate, IPSS - international prostate symptom score, CTCAE common terminology criteria for adverse events

\section{Declarations}

Ethics approval and consent to participate: This is an IRB approved study.

Consent for publication: All the patients in this study had consent obtained to use their data for publication at the time of radiation consent.

Availability of data and materials: The datasets generated and/or analyzed during the current study are not publicly available because it is continuously being updated with new cases, but is available from the corresponding author on reasonable request.

Competing interests: No competing interests declared by any of the authors.

Funding: Not applicable.

Author contributions: JP and DS contributed to gathering the data and writing the manuscript. LMG was responsible for creating the treatment plans. KJ was responsible for image gathering necessary for the study. KY is the prostate radiation expert who provided guidance and approved plans used in this study. NP is the corresponding author.

Acknowledgments: Editorial assistance was provided by the Moffitt Cancer Center's Scientific Editing Department by Dr. Paul Fletcher \& Daley Drucker. No compensation was given beyond their regular salaries.

\section{Citations}


1. Bechis SK, Otsetov AG, Ge R, Olumi AF. Personalized medicine for the management of benign prostatic hyperplasia. J Urol. 2014;192(1):16-23.

2. Rassweiler J, Teber D, Kuntz R, Hofmann R. Complications of transurethral resection of the prostate (TURP)-incidence, management, and prevention. Eur Urol. 2006;50(5):969-979; discussion 980.

3. Foster HE, Barry MJ, Dahm P, et al. Surgical Management of Lower Urinary Tract Symptoms Attributed to Benign Prostatic Hyperplasia: AUA Guideline. J Urol. 2018;200(3):612-619.

4. de Assis AM, Moreira AM, de Paula Rodrigues VC, et al. Prostatic artery embolization for treatment of benign prostatic hyperplasia in patients with prostates > 90 g: a prospective single-center study. J Vasc Interv Radiol. 2015;26(1):87-93.

5. Wang MQ, Guo LP, Zhang GD, et al. Prostatic arterial embolization for the treatment of lower urinary tract symptoms due to large (>80 $\mathrm{mL}$ ) benign prostatic hyperplasia: results of midterm follow-up from Chinese population. BMC Urol. 2015;15:33.

6. Uflacker A, Haskal ZJ, Bilhim T, Patrie J, Huber T, Pisco JM. Meta-Analysis of Prostatic Artery Embolization for Benign Prostatic Hyperplasia. J Vasc Interv Radiol. 2016;27(11):1686-1697 e1688.

7. Abt D, Hechelhammer L, Mullhaupt G, et al. Comparison of prostatic artery embolisation (PAE) versus transurethral resection of the prostate (TURP) for benign prostatic hyperplasia: randomised, open label, non-inferiority trial. BMJ. 2018;361:k2338.

8. Gao YA, Huang Y, Zhang R, et al. Benign prostatic hyperplasia: prostatic arterial embolization versus transurethral resection of the prostate--a prospective, randomized, and controlled clinical trial. Radiology. 2014;270(3):920-928.

9. Insausti I, Saez de Ocariz A, Galbete A, et al. Randomized Comparison of Prostatic Artery Embolization versus Transurethral Resection of the Prostate for Treatment of Benign Prostatic Hyperplasia. J Vasc Interv Radiol. 2020;31(6):882-890.

10. Pisco JM, Bilhim T, Costa NV, et al. Randomised Clinical Trial of Prostatic Artery Embolisation Versus a Sham Procedure for Benign Prostatic Hyperplasia. Eur Urol. 2020;77(3):354-362.

11. Malling B, Roder MA, Lindh M, Frevert S, Brasso K, Lonn L. Palliative Prostate Artery Embolization for Prostate Cancer: A Case Series. Cardiovasc Intervent Radiol. 2019;42(10):1405-1412.

12. Mordasini L, Hechelhammer L, Diener PA, et al. Prostatic Artery Embolization in the Treatment of Localized Prostate Cancer: A Bicentric Prospective Proof-of-Concept Study of 12 Patients. J Vasc Interv Radiol. 2018;29(5):589-597.

13. Ghadjar P, Vock J, Vetterli D, et al. Acute and late toxicity in prostate cancer patients treated by dose escalated intensity modulated radiation therapy and organ tracking. Radiat Oncol. 2008;3:35.

14. Yamazaki H, Nakamura S, Nishimura T, et al. Transitioning from conventional radiotherapy to intensity-modulated radiotherapy for localized prostate cancer: changing focus from rectal bleeding to detailed quality of life analysis. J Radiat Res. 2014;55(6):1033-1047.

15. Wu XZ, Xie GR, Chen D. Hypoxia and hepatocellular carcinoma: The therapeutic target for hepatocellular carcinoma. J Gastroenterol Hepatol. 2007;22(8):1178-1182.

\section{Figures}




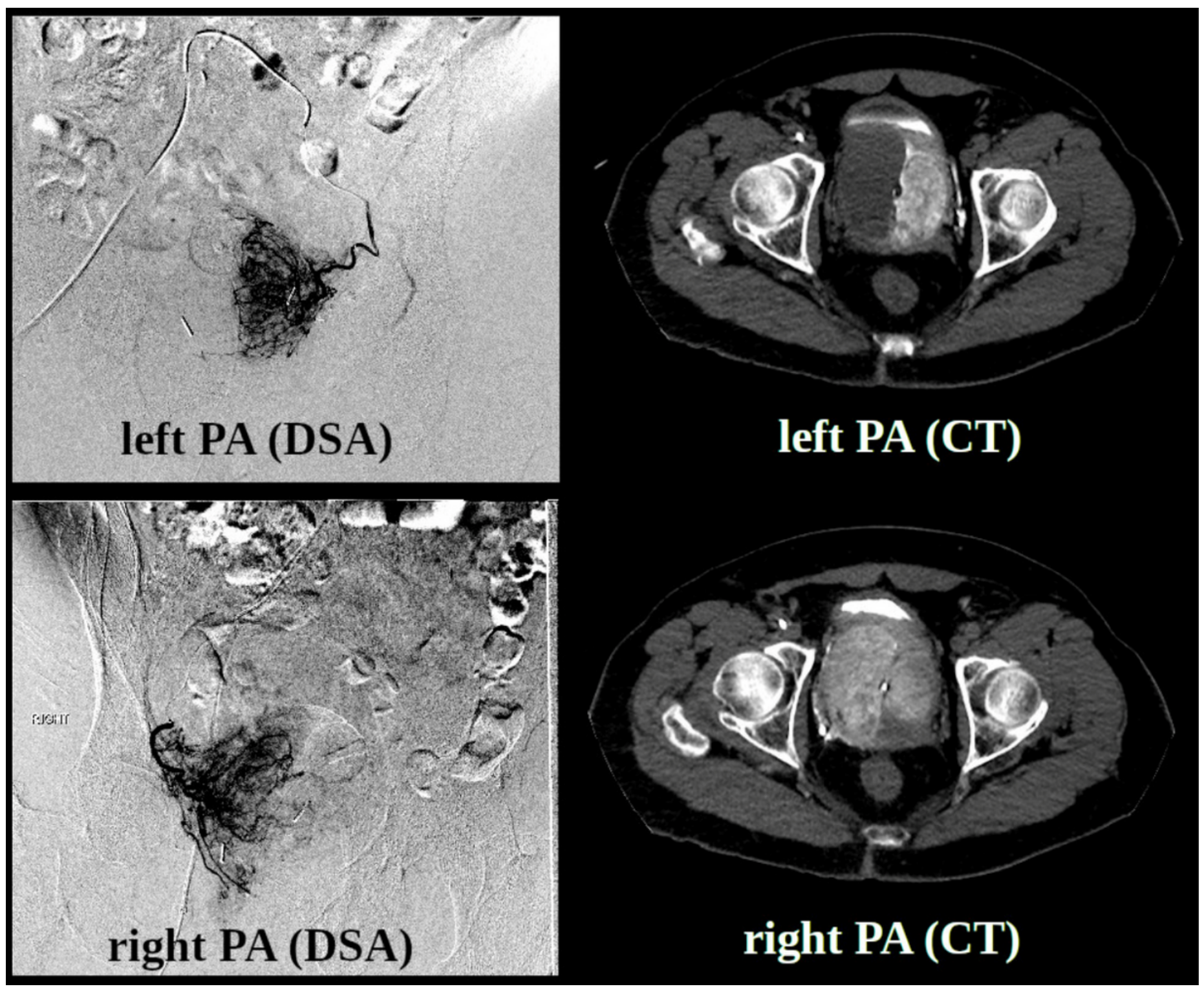

Figure 1

Superselective DSA (first column) and CT angiographic (second column) acquisitions of the left (first row) and right (second row) prostatic artery
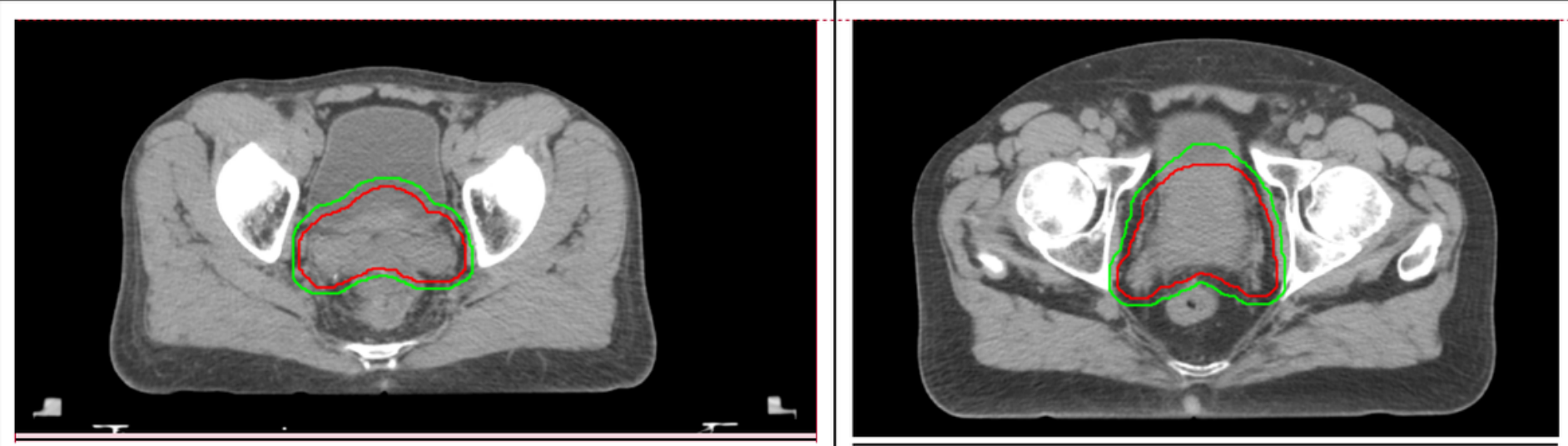

Figure 2 


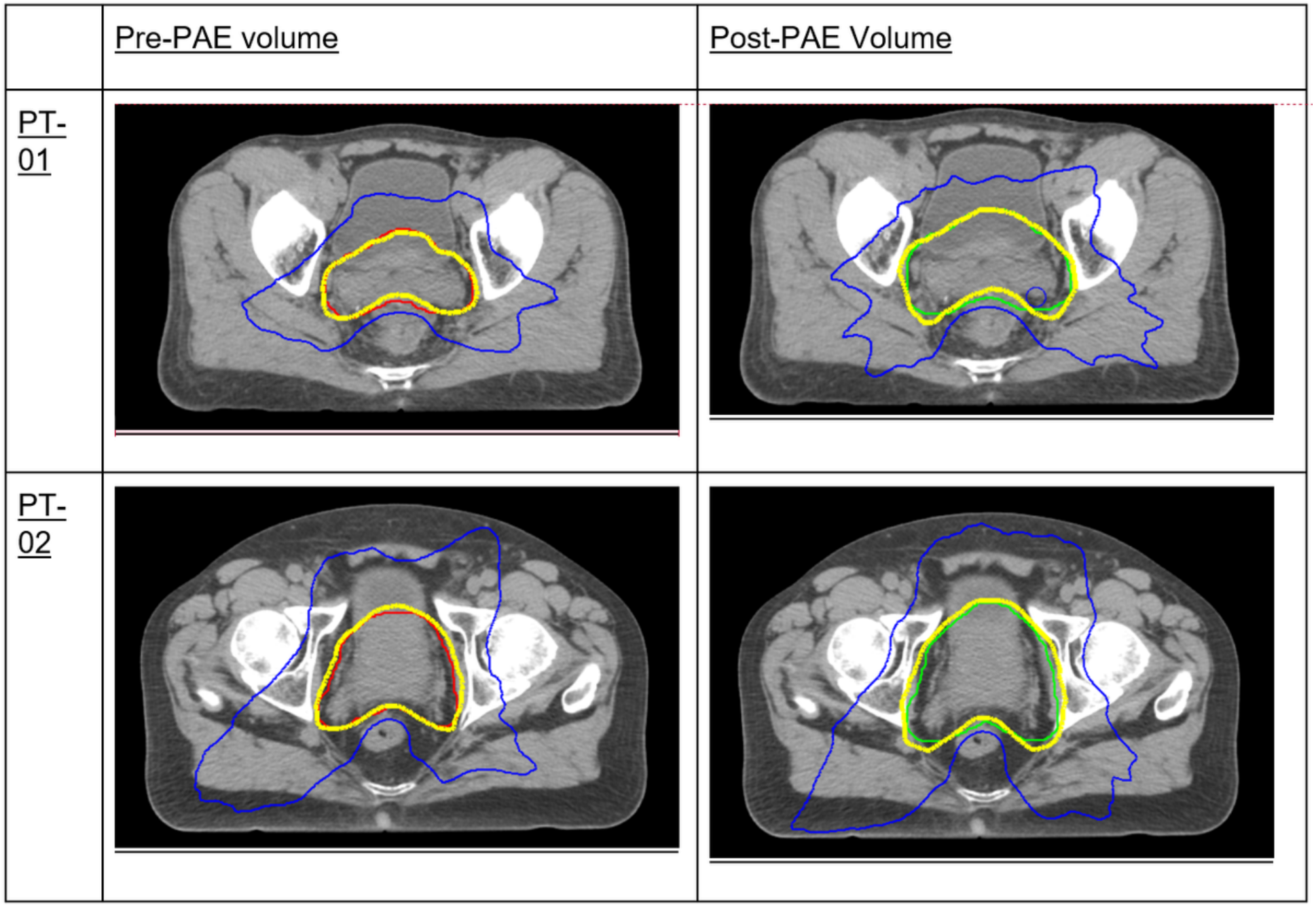

\section{Figure 3}

Top row is patient 1 treated to $60 \mathrm{~Gy}$ in 30 fractions. Bottom row is patient 2 treated to $78 \mathrm{~Gy}$ in 39 fractions. Two plans created using the prePAE volume (green) and post-PAE volume (red) PTVs. The yellow represents the prescription dose isodose line while the blue represents the $50 \%$ prescription isodose line. 